\title{
From the Editors' Desk: Realizing the Dream: Mentorship in Academic Medicine
}

\author{
Mitchell D. Feldman, MD, MPhil \\ Division of General Internal Medicine, Department of Medicine, University of California, San Francisco, San Francisco, CA, USA.
}

J Gen Intern Med 27(1):1-2

DOI: $10.1007 / \mathrm{s} 11606-011-1923-2$

(c) Society of General Internal Medicine 2011

I $\mathrm{n}$ his book on adult development first published in 1978, Daniel Levinson wrote that: "The mentoring relationship is 'one of the most complex and developmentally important' in a persons life." The mentor, Levinson said, will..."assist and facilitate the realization of the dream." While the business community early on recognized the critical role of mentoring in the professional accomplishments of its employees (most of my early education on mentoring came from papers published in the Harvard Business Review, not core medical journals), it is only over the past several years that there has been increasing interest in the importance of mentoring for career success and satisfaction in academic medicine. Research has found that faculty members across academic medicine who identify mentors are more productive and more likely to be promoted than their peers without mentors. Mentored faculty members are also more likely to obtain research funding and publish more papers and are less likely to leave their current institution.

Leaders in academic medical centers have taken note, and some have established new mentoring programs that aim to address issues of faculty career satisfaction, retention and productivity. At the University of California, San Francisco (UCSF), for example, a recently established campus-wide faculty mentoring program as well as a Mentor Development Program under the auspices of the Clinical and Translational Research Institute (CTSI) provide career and research guidance and mentor training for junior and mid-level faculty members. In addition, faculty members at UCSF are required to document mentoring on their $\mathrm{CV}$ so it can be considered as part of the promotion package. This is an innovation that all academic medical institutions should consider adopting.

But while mentoring has only recently been integrated into the fabric of academic medical education and training, mentoring is not a new concept, and despite the frequent references to Odysseus and Telemachus in the mentoring literature, it is not an exclusively western idea. In fact, mentoring relationships seem to be intrinsic to most types of social organizations and are present in most if not all cultures. For example, in Japan, while there is no exact translation of the word "mentor," mentoring is a central component of many work relationships both within and outside of medicine. In sumo wrestling, for example, there is a traditional mentoring system of sorts that is adhered to by all that live in the heya, or

Published online November 16, 2011 sumo training stable. The lowest rank of wrestler known as rikishi is responsible for cooking, preparing the bath, and even holding a towel for the sekitori, or higher ranked wrestler. A rikishi is expected to respect and obey their sekitori, and the sekitori in turn must guide, protect and teach their rikishi. While this system with its reliance on a strict hierarchy and subservience of the junior protégé to their senior sekitori would not work in the west, (I can't recall the last time one of my mentees prepared my lunch), it works well as a functional mentoring system to help train young sumo wrestlers in the art and techniques of their chosen profession.

Mentoring relationships have also been central to medical training in Japan. In the Japanese medical model, originally adapted from Germany, the oben (from the German for "at the top" or "above") is in charge of the medical or surgical team and is responsible for teaching and mentoring the junior kobun (or mentee). When this system works well, the senior physician (oben) will teach and protect the student and resident trainees on the team while expecting them to work hard and respect his authority, similar to the relationship between the sumo sekitori and the rikishi. The system may break down, however, if there is not good personal 'chemistry' between the oben (mentor) and kobun (mentee), as it is not socially or organizationally acceptable for the mentee to find a new oben.

As Rachel Hammer points out in her lovely essay about mentoring, "From the Shade into the Sun: In Search of a Mentor," featured in this month's Healing Arts, chemistry is the ineffable but crucial ingredient in most successful mentoring relationships. She writes: "A good match made between mentor and mentee is a romance, indeed. A curious chemistry...Mentoring relationships are critical in the cultivation of healthy, fruitful doctors. Eden born, I idealize. I wish the gardens were better organized; the fertilizer and soil more fairly distributed so there weren't so many forlorn shoots among the grateful sprouts."

Mentoring relationships, both formal and informal, albeit less hierarchical than is the rule in Japanese medical training, are becoming part of the fabric of academic internal medicine in the United States. In this issue of JGIM, Reid et al. examine mentorship, academic productivity and promotion among academic hospitalists in the US. They report on results of a web-based survey of 420 hospitalists and found that while most felt that having a mentor was important in academic career development, less than half reported having a current mentor. Further, they found that clinician-investigators were more likely to have mentors than clinician-educators, and that those with mentors were more likely to have publications (both peer and non-peer reviewed) and more likely to have led a session at a national meeting. The discrepancy in mentorship between clinician-investigators and educators has been 
reported in previous surveys and is a critical issue that must be addressed through the establishment of formal mentoring programs and mentor training for clinician-educator faculty.

In the spirit of mentorship, JGIM is pleased to be offering a new opportunity for senior GIM research fellows and junior GIM faculty interested in medical journalism. The JGIM Editorial Fellowship is a 1-2-year position in which fellows will work closely with a mentor to acquire skills in manuscript review, publication standards and all phases of manuscript adjudication. Visit this web page for more information on the program: http://www.sgim.org/index.cfm?pageId=325

Conflict of Interest: None disclosed.

Corresponding Author: Mitchell D. Feldman, MD, MPhil; Division of General Internal Medicine, Department of Medicine, University of California, San Francisco, 1545 Divisadero, San Francisco, CA 94143-0320, USA (e-mail: mfeldman@medicine.ucsf.edu). 